\title{
Concept of large scale PV-WT-PSH energy sources coupled with the national power system
}

\author{
Jakub Jurasz ${ }^{1, *}$, Jerzy Mikulik ${ }^{1}$, Magdalena Krzywda ${ }^{1}$ \\ ${ }^{1}$ AGH University of Science and Technology, 30 Mickiewicza Ave., 30-059 Kraków, Poland
}

\begin{abstract}
Intermittent/non-dispatchable energy sources are characterized by a significant variation of their energy yield over time. In majority of cases their role in energy systems is marginalized. However, even in Poland which is strongly dedicated to its hard and brown coal fired power plants, the wind generation in terms of installed capacity starts to play a significant role. This paper briefly introduces a concept of wind (WT) and solar (PV) powered pumped storage hydroelectricity (PSH) which seems to be a viable option for solving the problem of the variable nature of PV and WT generation. Additionally we summarize the results of our so far conducted research on the integration of variable renewable energy sources (VRES) to the energy systems and present conclusions which strictly refer to the prospects of large scale PV-WT-PSH operating as a part of the polish energy system.
\end{abstract}

\section{Introduction}

Accordingly to the recently published reports [1] and scientific analysis [2] the world has to urgently and rapidly transfer its energy system from the power plants fueled by finite energy resources to that based on renewable energy sources (RES) which are perceived as one of the solutions which can impede the climate change and its ominous consequences.

Historically, the RES where implemented as testing facilities for scientific purposes or were used in harsh terrain and conditions where the solutions based on conventional energy sources were not sufficient or completely not applicable. What is interesting, in the past large financial investments in the RES development were made mainly by the oil and gas industry [3,4]. It is the oil and gas companies found photovoltaics and wind turbines as a viable energy sources in remote areas to power radio transmitters situated along the transmission line or for cathodic protection of pipes. Some even consider them as a suitable energy source for powering the autonomous pumping rigs.

On the large scale, the solar and wind energy sources firstly became popular on the islands. Especially those located far from the mainland and not on the main maritime transport routs. The main reasons for their popularity lie behind the: lack of natural energy resources on the islands which would be sufficient to cover the energy needs; usually favorable wind and/or solar resources for their development as well as necessary height

\footnotetext{
*Corresponding author: jakubkamiljurasz@gmail.com
} 
differences to create pumped storage-hydroelectricity energy storing facilities; need to transport fuel from the mainland in from of usually heavy oil or coal to power conventional power plants what in consequence leads to a greater local pollution as well as decreases the society energy security as well as increase the energy costs.

However, the variable, intermittent, predictable to a limited extent and non-dispatchable nature of solar and wind energy sources makes their utilization an arduous task. Additionally their availability is not always (if at all) positively correlated with the energy demand [5]. But it is important to underlie that in case of Poland the changing patterns of the energy demand and their relation to the observed mean temperatures [7] which in turn are related to the irradiation values which affect determine the energy yield from PVs creates a market niche for solar powered air-conditioners [8].

An obvious solution to the problem of the intermittency of solar and wind resources is to couple PV and WT energy sources with energy storing device. But still even if energy storage will be added it is important to use both energy generating technologies because of their beneficial temporal complementarity which especially exhibits itself on an annual time scale $[9,10]$. The impact of using two or more complementary energy sources usually decreases the need for energy storage and makes the energy source more reliable. On a small scale such hybrids will be utilizing battery banks in from of e.g. lithium-ion batteries, whereas on a larger a pumped storage-hydroelectricity will be implemented. It is important to underlie that the PSH is the most mature and almost the only bulk energy storage technology available today. Some substitute technologies have been developed such as compressed air energy storage (CAES) [11,12] however, they not have been implemented on a large scale, two facilities are operating in Huntorf (Germany) and McIntosh (USA).

In this paper we will describe the basic concepts behind the operation of the wind and solar powered pumped storage-hydroelectricity operating as a part of the national power system. Drawn conclusions will be based on the calculations conducted in the so far conducted research available in $[5,13]$.

\section{Conceptual design of the PV-WT-PSH}

A thorough analysis of the existing and proposed concepts of the PV/WT-PSH hybrids was made in $[5,13]$. Therefore we will refrain ourselves from referencing those works once again and concentrate on describing the basic principles of such energy source operation and its impact on the national power system (NPS).

As already mentioned PV-WT-PSH hybrids consists of two non-dispatchable energy sources in form of photovoltaics which energy yield depends respectively on the current irradiation/irradiance and wind turbines which blades are moved by the flowing air masses and the propel the generator which in turns generate electricity. To some extent in case of PVs an assumption can be made that the energy yield is linear to the value of momentarily available irradiation, whereas in cases of WTs the energy output is strongly dependent on the wind speed to the power of three - naturally those characteristics are a significant simplification. Important aspects which should be also underlined include: the temperature impact of the PV cells efficiency and the fact that each wind turbine is represented by its specific power curve. In general the WT turbine power curve can be described by three characteristic wind speeds. Namely: $\mathrm{v}^{\text {cut-in }}-$ is a wind speed at which the WT starts to generate electricity, $v^{\text {rated }}$ - is a wind speed at which WT starts to generate electricity with its rated capacity, $\mathrm{v}^{\text {cut-off }}$ - is a wind speed at which the WT must be shot down for safety reasons. Commercially available large scale wind turbines manufactured by Vestas, Gamesa or Enercon usually start to generate electricity when the wind speed is greater than $4 \mathrm{~m} / \mathrm{s}$ and reach their rated power output for winds exceeding $12 \mathrm{~m} / \mathrm{s}$ but no faster than 
$25 \mathrm{~m} / \mathrm{s}$. Those two energy sources cannot be controlled and their energy/power output can be predicted with a certain margin for error and for a limited time horizon [14, 15].

The second part of the considered hybrid energy source is the pumped storagehydroelectricity. It is a relatively old concept. The first PSH went into the operation in 1909 at Schaffhausen in Switzerland and is still in operation [16]. Each PSH facility consists of two reservoirs. It is important that those reservoirs are close to each other but there remains a significant elevation difference between their water levels. This elevation difference is crucial because the potential (storing capacity and power output) of the PSH are mainly determined based on the basic equation determining the potential energy $E=m g h$, where: $\mathrm{E}-$ is the potential energy $[\mathrm{J}], \mathrm{m}-$ is the mass $[\mathrm{kg}], \mathrm{g}-$ is the gravitational acceleration $\left[\mathrm{m} / \mathrm{s}^{2}\right], \mathrm{h}-$ is the mentioned height difference $[\mathrm{m}]$. The brief analysis of the above reminded equations indicates that the potential of given site for the PSH facility development will be to the same extent determined by the available water storing capacity of the upper and lower reservoir as well as the elevation difference between reservoirs. It is important to stress that both conditions must be met to given site be further considered for the PSH development. Naturally the subsequent analysis of the site predisposition for the PSH project will consider other criteria such as environmental impact, closeness to the transmission lines, amount and complexity of soil works etc.

Initially the PSH facilities were erected in order to compensate for the intra-day variability of energy demand and supply. It is clear that during night the energy demand is relatively lower than over daylight. Therefore the supply side must adjust to the occurring demand (please bear in mind that the concepts of the demand side management (DSM) are relatively novel and gained on popularity with the massive advent on VRES). So basically it is the conventional power plants that have to adjust their power output to meet the demand. But unfortunately, the conventional power plants are designed to operate within given range of parameters e.g. power output for which their efficiency is maximal and simultaneously the emissions are minimal. What is more sudden ramps up and down of the conventional power plants in terms of their power output does not positively impact their life span, reliability and economics.

The proposed almost two centuries ago solution is similar to that common on the other goods market. Meaning one has store the occurring energy surpluses and release them when the demand will occur. Naturally this solution is associated with additional questions like what is the optimal potential of the storage facility, how much energy can be freed per unit of time, what is the efficiency of the whole process, are there any loses if the storing period will elongate? We will address those questions in following paragraphs.

How this solution will work in case of the PSH facility? First of all the energy demand is smaller over the night. Some conventional power plants can curtail their power output but it is not possible in case of all of them. Therefore on the energy market occurs an energy surplus, meaning that the supply is greater than the demand and in consequence the energy price falls. Here comes the opportunity for the energy storing facilities in form of the PSH. They buy and then use that surplus energy to power the water pumps and force the flow of water from the lower reservoir to the upper one. Naturally some losses occur due to the non-perfect efficiency of the pumps. The volume of energy which can be pumped to the upper reservoir is determined by the pumps throughput and the reservoir available capacity. The night is over and a new day starts. People turn on lights, electric kettles and prepare for work. Offices and other businesses start their normal activities. The energy demand starts to pick up. It is followed by conventional power plants adjusting their power output. But they not always are able to keep up the pace. The situation on the energy market changes and now the energy demand is greater than the supply. In consequence the energy prices goes up. Here comes the opportunity for the PSH facilities which are characterized by the relatively fast reaction time. The during night pumped water to the upper reservoir can be 
now released back to the lower reservoir and its potential energy can be converted in water turbines into the electricity. The decisions whether the water from the upper reservoir should be discharged must be based on the difference between the cost at which it was pumped and the potential profit from discharging it and selling - especially in case when such facility operates on the energy market and should bring revenue.

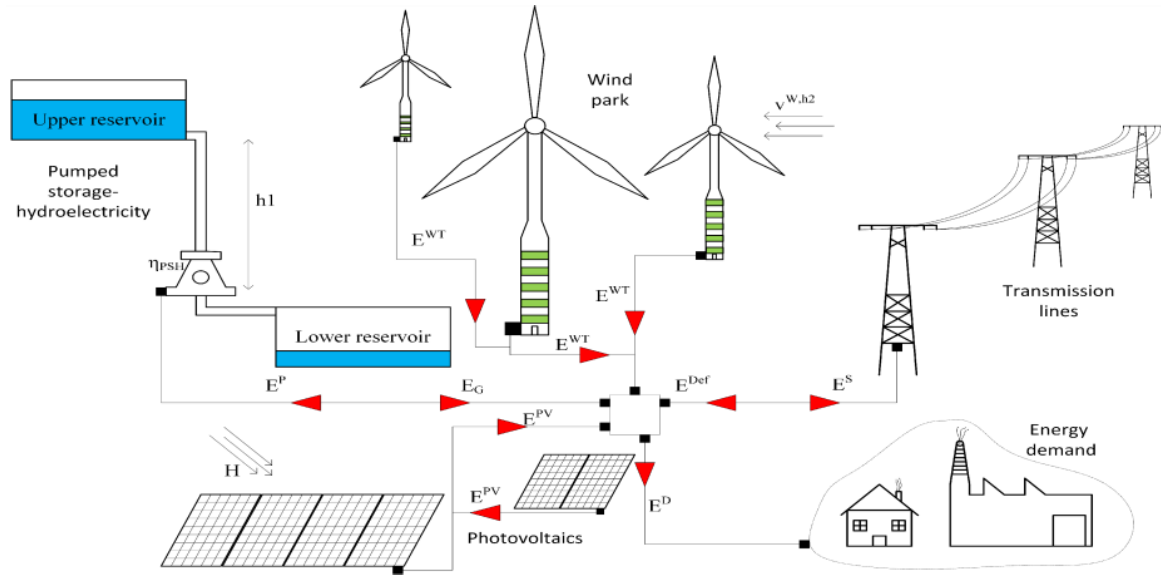

Fig. 1. Conceptual design of a grid connected PV-WT-PSH hybrid energy source.

Figure 1 visualizes the general concept of wind and solar powered PSH. As can be seen it is coupled to the energy demand source (here represented by a single house and a factory) and to the transmission lines. In the considered case the PSH consist of a single water turbine which can generate electricity or pump water from the lower to the upper reservoir (store electricity in a form of the potential energy). The water turbine is represented by its efficiency $\left(\eta^{\mathrm{PSH}}\right)$ which usually varies from 80 to $90 \%$ and its maximal power capacity. The capacity of the upper and lower reservoir is given in thousands of cubic meters but can be easily changed into energy units by considering the elevation difference between reservoirs (h1). In case of the non-dispatchable energy sources we have denoted to characteristic parameters which in the simulation models were used as hourly time series, namely: irradiation $(\mathrm{H})$ and wind speed $\left(\mathrm{v}^{\mathrm{h} 2}\right)$ which is observed at the wind turbine rotor height $(\mathrm{h} 2)$.

On the above presented figure, red dots indicate the possible energy flow directions. Naturally, from the energy sources $\left(\mathrm{E}^{\mathrm{WT}} \& \mathrm{E}^{\mathrm{PV}}\right)$ the energy flows only in one directions - they do not exhibit any self-consumption. An opposite situation can be observed in case of the energy consumers - they only exhibit the energy demand $\left(E^{D}\right)$ - in reality some of the energy consumers may be also utilizing their own energy sources such as PV and WT and be so called prosumers, however for the simplicity of calculations we put aside this possibility. The energy flow in both directions is possible in case of the PSH and the transmission lines. In the first case we have a volume of energy which was used to pump the water to the upper reservoir $\left(E^{P}\right)$ and the volume of energy generated $\left(E^{G}\right)$ by discharging the water from the upper to the lower reservoir. In the best case scenario to PV, WT and the PSH should be able to cover the entire energy demand without generating energy surpluses. An energy surplus in case of such system which is connected to the transmission lines is understood as an energy which was generated from PVs and WTs and after using some part of it to cover the energy demand, the remaining part could not be stored in the upper reservoir, due to its limited capacity or the curtailed throughput of the water turbine. Naturally, an opposite situation may occur when then available energy from PVs and WTs and that stored in the upper reservoir will not be sufficient to cover the energy demand. In such circumstances there will be a need to cover this energy deficit from the grid $\left(\mathrm{E}^{\text {Def }}\right)$. 
Energy deficits and surpluses have a random nature. It means that there will be a unpredictable energy flow between the PV-WT-PSH and the transmission lines (the energy system). Such situation is not desirable by the power system operators. They will have to adjust the power output of the conventional power plants with regard to the changing energy flow and direction. Therefore the PV-WT-PSH hybrid energy source should be sized in such a manner that the total sum of energy exchange with the power system is minimal. The best situation will be when the PV-WT-PSH would be able to guarantee the total energy self-sufficiency. This is theoretically possible but such system must be able to deal with extreme situations, like:

- Over prolonged time period the wind turbines and the photovoltaic installations are operating with their almost maximal power capacities. In consequence there occur a significant energy excess which must be stored in the upper reservoir. However, the energy storing capacity of the PSH is strongly limited and after several dozen hours or days there is no more possible to store there the energy from WT and PV which has not been used to cover the energy demand;

- A contrary case is when the energy from WT and PV is not sufficient to cover the energy demand. In consequence the volume of energy stored in the PSH is slowly declining. If the unfavorable weather conditions (low wind speed and irradiation) will prevail for next several hours or days the energy stored in the PSH will soon run out and the energy deficit will occur.

Even if such situation happen very rarely or on a lesser scale it will be still mandatory to oversize the whole system. By oversizing one should understood adding additional capacity installed in PVs and WTs. In general the energy generated by those both sources should be greater than the energy demand augmented by the losses occurring during the energy storing processes (not perfect efficiency of the water turbines). However, some additional capacity seems to be mandatory due to the long term variability of the mean annual wind speeds and general tendencies which indicate that the cumulative energy demands picks up. To sum up, the energy storage facility should be able to overcome the problem of the variable nature of the wind and solar energy sources. It is important to underlie that this problem may be overcome by coupling several such hybrid energy sources. It is however required that they must be relatively far apart if one wants to makes use of the spatial complementarity phenomena exhibited by some of the VRES. Spatial complementarity works similar to the temporal complementarity and it can be applied to the energy sources using the same energy resource e.g. wind parks located in two different areas. The impact of spatial complementarity is usually known as smoothing the energy generation curve by spatial distribution of variable energy sources $[14,16]$. The above described problem of the stochastic nature of the volumes and directions of the energy exchange with the grid has been addressed in [5] and the presented there conclusions indicate that to some extent it can be solved by applying adequate forecasts. Meaning that it will be hard to eliminate those energy flows by at least it is possible to predict them. Naturally some error will occur but they depend on the quality of the forecasting models.

\section{PV-WT-PSH operation}

We have already mentioned that the PV-WT-PSH hybrid energy source may aim at ensuring the energy autarky. This is especially a case in the isolated energy systems like those observed on the islands. However, here we consider them as a part of the larger energy system with all its positive and negative consequences. On the Fig. 2 we have visualized the operation on such system in situation when it should cover the whole energy demand. This is just an exemplar day which might have occurred. Please note the purple and squared area which indicate the unscheduled energy exchange with the grid. On the 
Fig. 2-4 we have purposefully omitted the pumping operation of the PSH.

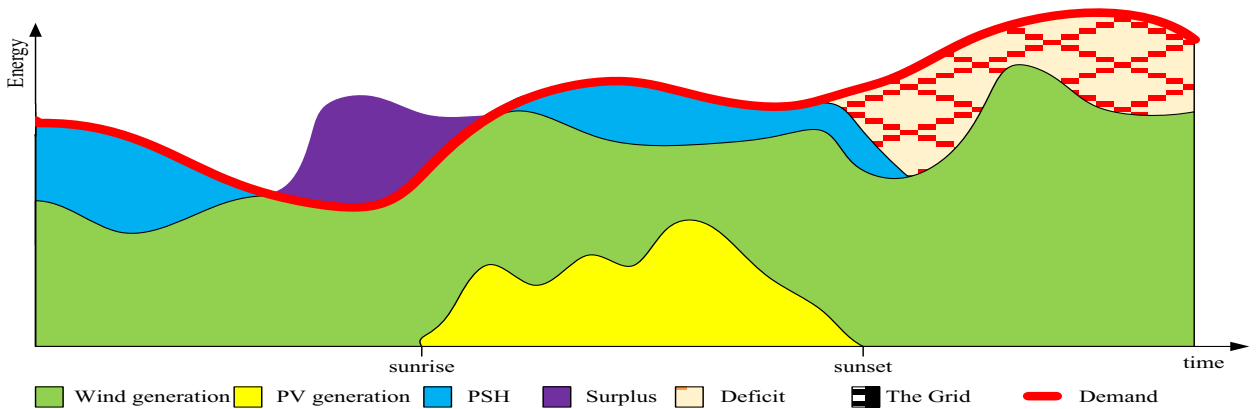

Fig. 2. The theoretical PV-WT-PSH operation assuming that some load is covered from the grid.

Because ensuring the energy self-sufficiency whilst minimizing the occurrence of the energy surpluses is a complicated and in some cases almost an impossible task we suggest two additional scenarios. On the Fig. 3 and 4 one can observe that some part (what is important a fixed value) of the energy demand is being covered from the grid (black squared area). The basic assumption which lies behind the scenario presented on the Fig. 3 is such that some part of the demand will be covered from the grid when there is no possibility to generate electricity from the PV installation. In general organizing hybrid energy system operation according to the theoretical energy yield from the PVs is a simpler task. This results from the fact that it is possible to exactly determine when they should generate energy. This is done based on the clear-sky models. Therefore, for the each day of the year one can establish precise hours when some volume of the energy should be delivered from the grid/energy system to cover the energy needs of the customers using the PV-WT-PSH hybrid. As shown in [13] such solution should not only ease the sizing procedure of the mentioned hybrid energy source but also minimize the volume of the unscheduled energy exchanges with the energy system. What is more, the values of the scheduled energy exchange (on the Fig. 3-4: The Grid) can vary for different parts of the year accordingly to the changing availability of the wind energy.

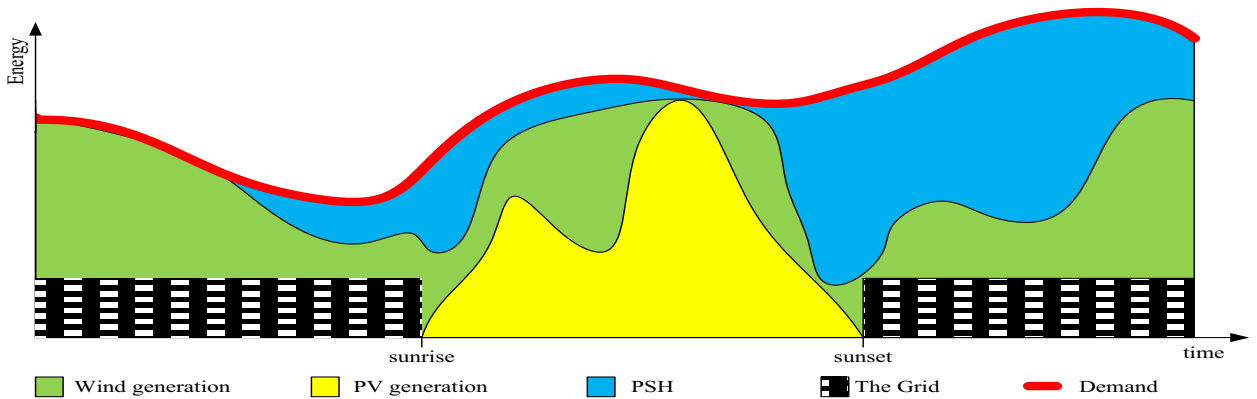

Fig. 3. The theoretical PV-WT-PSH operation assuming that some load is covered from the grid.

If the solution presented on Fig. 3 is not sufficient it is always possible to establish a fixed volume of the energy demand which should be covered from the energy system (see Fig. 4). This is also a beneficial situation because the power system will be dealing only with the so called base load (an energy demand which does not change and is observed over the whole year). In consequence the PV-WT-PSH will have to cover relatively smaller demand. The main benefit of such approach is such that the unscheduled energy exchanges can be significantly reduced and the from the perspective of the national power system operator the energy demand of the selected group of energy consumers is seen as a fixed 
value. Therefore, it is easy to schedule the operation of the conventional power plants. What is more such load can be covered from power plants which generate the electricity with the lowest costs.

Naturally, many other scenarios are possible. For example: the fixed value of energy delivered from the grid can vary for each hour of the day; the system operator would see it as very promising when the PV-WT-PSH will be used to perform so called peak shaving what boils down to covering the energy demand it tends to exhibit the highest values (morning and evening energy demand peak).

With the advent of the variable renewable energy sources in the power systems researchers are searching for various approaches and solutions which can ease the process of their integration. Also from the perspective of the mentioned PV-WT-PSH hybrid a very promising tool is the so called demand side management (DSM) which may be able to slightly reduce the occurrence of energy surpluses and deficits [18]. An important aspect is also in the form of the privately owned energy sources like: small scale PV installations and wind turbines coupled with the battery banks. Such relatively small energy sources may also enable smoother and more beneficial for the power system operation of the PV-WT-PSH hybrid.

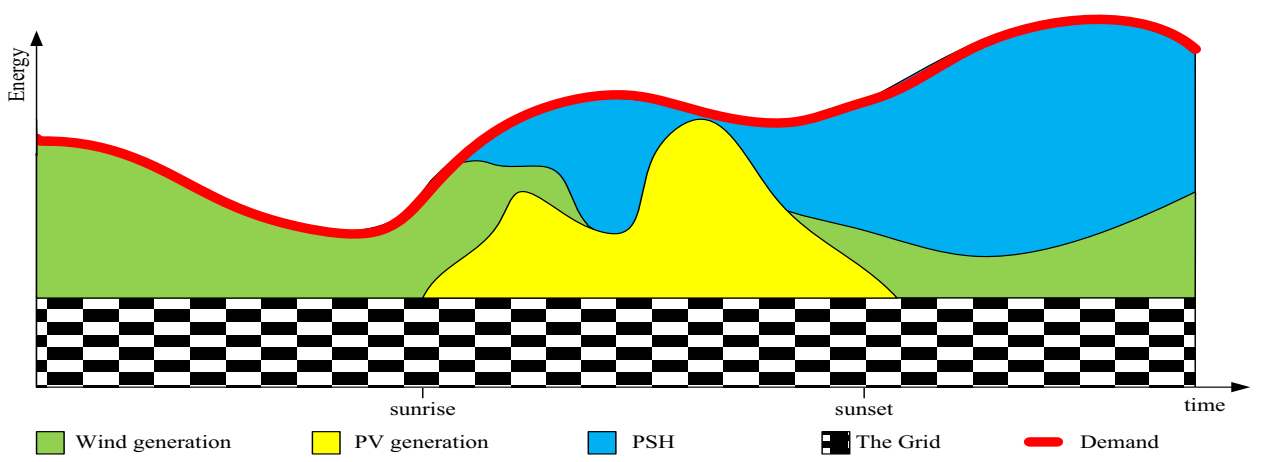

Fig. 4. The theoretical PV-WT-PSH operation assuming that some load is covered from the grid.

An open question remains about the possibility of creating such hybrid energy sources in the existing energy systems. As it has been already mentioned the PSH facilities were necessary to overcome the problem of the changing energy demand. In case of the PV-WT-PSH they would be also applied to compensate for the varying generation of nondispatchable solar and wind energy sources.

The polish energy system has five operating PSH stations. The sixth which was commissioned in 1997 is currently operating as run-off-river power plant with pondage. One of the PSHs located in Żarnowiec (Northern Poland) was designed to cooperate with the first polish nuclear power plant but following governments delayed this project and its perspectives are murky. The biggest problem when it comes to the PSH facilities is finding a suitable location which will ensure sufficient height difference between reservoirs. Additional criteria which are usually used eliminate majority of the potential sites. The conducted in [13] literature review indicated that the cumulative capacity of the notrealized PSH projects in Poland amounts to $5.3 \mathrm{GW}$.

Summarizing above described potential of the PSH plants in Poland it is important to underlie that those projects are relatively old. It seems that due to the changing environmental regulations some of them could not be realized today. However, it is clear that Poland has significant potential for developing PSH facilities on a large scale. The described in this paper concept can be applied for much smaller PSH stations which combined with the PVs and WTs will be used to cover the energy needs of smaller communes: like villages or small towns. 


\section{Summary}

The PSH stations coupled with the wind and solar energy sources are a common concepts known in the isolated energy systems located on islands. In this short postconference publication we have briefly introduced this concept on the background on our recent works in area of the integration of variable renewable energy sources to the power system. Presented overview aims at: familiarizing the potential leader with those concepts; summarizing the general results of our works and to initiate the debate on the possibility of finding market niches for such solution in the existing and the future energy systems where RES will undoubtedly play a significant role.

\section{References}

1. S. A. Crate, \& M. Nuttall, (Eds.). Anthropology and climate change: from encounters to actions (Routledge, 2016)

2. J. Hansen, P. Kharecha, M. Sato, F. Ackerman, D. Beerling, P Hearty. J., E. J. Rohling. PLoS One, 8(12), e81648 (2013)

3. J. Wang, J. O'Donnell, A. R. Brandt, Energy 118 (2017)

4. G. S. M. Teale, Journal of petroleum technology 36, 05 (1984)

5. J. Jurasz. Energy Conversion and Management 136 (2017)

6. J. Jurasz, \& J Mikulik. International Journal of Photoenergy (2016)

7. J. Jurasz, J. Mikulik, \& A. Piasecki, Przegląd Elektrotechniczny 92 (2016)

8. J. Jurasz, M. Krzywda, \& J. Mikulik. In E3S Web of Conferences (Vol. 10, p. 00059). EDP Sciences (2016)

9. J. Jurasz, A. Piasecki, \& M, Wdowikowski. In E3S Web of Conferences (Vol. 10, p. 00032). EDP Sciences (2016)

10. J. Jurasz, \& A. Piasecki. Acta Energetica 2, 27 (2016)

11. F. Crotogino, K. U. Mohmeyer, R. Scharf, Huntorf CAES: More than 20 years of successful operation (Orlando, Florida, USA, 2001)

12. J. Taylor, \& A Halnes. Analysis of compressed air energy storage. In PCIC Europe 2010 conference record (pp. 1-5). IEEE (2010)

13. J., Jurasz. Optimization of the installed capacity in the solar-wind-pumped storage hydroelectricity power source. (Unpublished $\mathrm{PhD}$ dissertation). AGH University, Cracow, Poland (2016)

14. J. Kleissl. Solar energy forecasting and resource assessment. Academic Press (2013)

15. M. Lei, L. Shiyan, J. Chuanwen, L. Hongling, \& Z. Yan. Renewable and Sustainable Energy Reviews 13, 4 (2009)

16. T. H. Douglas, (Ed.). Pumped Storage: Proceedings of the Conference Organized by the Institution of Civil Engineers at Imperial College of Science, Technology and Medicine, London on 2-4 April 1990. Thomas Telford (1990)

17. J. Jurasz, J. Mikulik, Rynek Instalacyjny 11 (2015)

18. M. R. Staats, , P. D. M. De Boer-Meulman, W. G. J. H. M. van Sark. Sustainable Energy, Grids and Networks (2016) 